\title{
Thresholding Approach to Radiography Image Processing Acceleration
}

\author{
Vladimir S. Ostojić, Đorđe S. Starčević, and Vladimir S. Petrović
}

\begin{abstract}
An approach for detecting low-value Laplacian pyramid coefficients which can be used to accelerate radiography image processing is presented. The acceleration is achieved through modifying Laplacian pyramid construction algorithm. Detection of low-value coefficients in lower pyramid layers is based on corresponding low-value higher layer coefficients which are lower than the threshold. Detected coefficients are then omitted in pyramid calculation and by the radiography image enhancement algorithm. Clinical radiography images are used to evaluate the proposed approach. Structural similarity index (SSIM) is employed to measure similarity between images processed with and without proposed acceleration. Threshold value analysis shows that high image quality with a mean SSIM of 0.995 can be achieved with mean processing time reduction of $4.62 \%$.
\end{abstract}

Keywords - digital radiography, image processing, Laplacian pyramid, multi-scale methods.

\section{INTRODUCTION}

$\mathrm{R}$ ADIOGRAPHY images are processed with the purpose of improving detail visibility, enhancing contrast, compressing a high dynamic value range, reducing noise, etc. Multi-scale approaches have been efficiently employed to address these issues [1]-[8].

Laplacian pyramid (LP) [9] was used in several proposed radiography image processing methods. Nonlinear mapping of LP coefficients for contrast amplification of medical images was presented in [1]. Another LP based approach for noise containment and contrast enhancement combined with local activity and density estimations was proposed in [2]. A nonlinear multi-scale contrast enhancement algorithm for chest radiographs based on local contrast

Paper received May 6, 2017; revised May 11, 2017; accepted May 30, 2017. Date of publication July 31, 2017. The associate editor coordinating the review of this manuscript and approving it for publication was Prof. Branimir Reljin.

This paper is a revised and expanded version of the paper presented at the 24th Telecommunications Forum TELFOR 2016 [15].

This research is supported by grants TR32040 and TR32035 of Serbian Ministry of Science.

Vladimir Ostojic is with the University of Novi Sad, Serbia, Faculty of Technical Sciences, Department of Power, Electronics and Telecommunications (e-mail: vladimir.ostojic@uns.ac.rs).

Đorđe Starčević is with the University of Novi Sad, Serbia, Faculty of Technical Sciences, Department of Power, Electronics and Telecommunications (e-mail: djordje.starcevic@uns.ac.rs).

Vladimir Petrović is with the University of Novi Sad, Serbia, Faculty of Technical Sciences, Department of Power, Electronics and Telecommunications (e-mail:vladimir.petrovic@uns.ac.rs). information was proposed in [3]. Wavelet transformation was used for contrast enhancement of mammographic images in [4] and chest radiographs in [5]. Comparison of Laplacian and wavelet pyramids with the purpose of contrast enhancement for radiography images was performed in [6]. It was shown that artifacts produced by nonlinear LP coefficient mapping are less prominent than the ones produced by discrete wavelet transformation. Fast multi-scale morphological operations for local contrast enhancement were proposed in [7]. Machine learning of multi-scale patches for noise removal in fluoroscopy was proposed in [8]. Multi-scale approaches for medical image denoising were proposed in [10]-[12].

Due to the fact that radiography images are large with respect to pixel count (ranging from 9 megapixels for diagnostic x-ray [13] up to 25 megapixels for digital mammography [14]), processing time reduction should be considered along with assuring the accomplishment of processing objectives. Therefore, in this paper we present a research into processing acceleration with the constraint of image quality preservation.

Employing LP over wavelet transform as a multi-scale technique was motivated by the fact that LP produces less prominent artifacts. We address the detection of low-value Laplacian pyramid coefficients and how it can be used for reducing processing time. Preliminary research into this topic was presented in [15].

In Section II we outline the radiography image enhancement algorithm in which the low-value Laplacian pyramid coefficient detection was embedded during evaluation. Laplacian pyramid and image reconstruction algorithms are explained in Section III. A method for detection of Laplacian pyramid coefficients with low-value is presented in Section IV. Modification of the pyramid algorithm achieved with the detection is outlined in Section $\mathrm{V}$. Analysis of the processing time reduction is made in Section VI. Algorithm evaluation and results discussion are presented in Section VII. Section VIII concludes the paper.

\section{RADIOGRAPHY IMAGE ENHANCEMENT}

In order to accomplish the outlined radiography image processing objectives, we chose to embed our proposed method into a processing algorithm, presented in [16]. Additional motivation behind the use of this algorithm is that it operates on LP coefficients, which is mutual with our research.

Detail visibility improvement and contrast enhancement are achieved through LP coefficients value manipulation. LP coefficients with a low absolute value are amplified in 
order to increase the visibility of fine details and improve local contrast. High absolute value LP coefficients are attenuated to additionally enhance image contrast. Both operations are performed using a single nonlinear mapping function with different parameters.

It was noted that very low absolute value LP coefficients which correspond to finer details represent noise. Noise reduction is achieved by avoiding these coefficients in the previously explained nonlinear mapping.

High dynamic value range compression is performed in the preprocessing stage, i.e. before LP decomposition of the unprocessed image, with linear to logarithmic domain conversion of pixel intensities. More details can be found in [16].

\section{LAPLACIAN PYRAMID}

LP is a representative of multi-scale approaches to image processing. It is composed of layers which represent bandpass images obtained in a dyadic manner. These band-pass images can be interpreted as representations of image details of different scales, which is utilized by enhancement algorithms as mentioned in Sections I and II. LP is closely related to the Gaussian pyramid, and they can be obtained through the use of the algorithm whose pseudo code is presented in Fig. 1.

\begin{tabular}{c} 
Input \\
$G P_{0} \leftarrow$ input image I \\
lpf $\leftarrow$ low-pass filter kernel \\
For $i=0 \ldots N-1$ \\
$G P_{i+1}=$ convolution $\left(G P_{i}\right.$, lpf $)$ \\
$G P_{i+1}=\operatorname{subsample}\left(G P_{i+1}\right)$ \\
$\widehat{G P}_{i}=$ upsample $\left(G P_{i+1}\right)$ \\
$\widehat{G P}_{i}=$ convolution $\left(\widehat{G P}_{i}, l p f\right)$ \\
LP $P_{i}=G P_{i}-\widehat{G P}_{i}$ \\
end \\
Res $=G P_{N}$ \\
\hline Fig. 1. Laplacian pyramid algorithm
\end{tabular}

Fig. 1. Laplacian pyramid algorithm.

In Fig. $1, N$ is the number of pyramid layers and the low-pass filter is denoted as $l p f$. More details on coefficient selection for this filter can be found in [9], [17] and [18]. In our research, we used a binomial filter proposed in [19] as it allows faster implementation.

$G P_{i}$ is a layer of the Gaussian pyramid. Each layer represents a low-pass approximation of the previous layer obtained after filtering and subsampling. Subsampling is achieved by removing every even column and row from the image, while upsampling is performed by adding zerovalued rows and columns between the existing ones. Last image in the Gaussian pyramid sequence is denoted as Res. $L P_{i}$ stands for a layer of the LP. Each $L P_{i}$ is a band-pass image which is determined as the difference between Gaussian pyramid layer $G P_{i}$ and upsampled and filtered layer $G P_{i+1}$.

Image reconstruction algorithm requires only LP and Res. A pseudo code for the reconstruction algorithm is presented in Fig. 2. Output of the reconstruction algorithm $\hat{I}$, which is the image Res after successive upsampling and filtering, corresponds to the original image if no changes were made to the LP or Res before the start of reconstruction.

\begin{tabular}{c} 
For $i=N-1 \ldots 0$ \\
Res $=$ upsample $($ Res $)$ \\
Res $=$ convolution $($ Res,$l p f)$ \\
Res $=$ Res $+L P_{i}$ \\
end \\
Output \\
$\hat{I} \leftarrow$ Res \\
\hline Fig. 2. Image reconstruction algorithm.
\end{tabular}

\section{COEFFICIENT DETECTION}

Radiography image can contain regions that do not depict anatomy. One such region is the image background, i.e. the region that is intentionally shielded from the irradiation source by the collimator, an integral part of radiography systems used to reduce the patient dose. Hence, the previously mentioned image region does not contain information relevant for the patient examination.

Another radiography image region that does not depict anatomy is the foreground corresponding to the directly irradiated part of the x-ray detector. In contrast to the background, which is the low-valued part of the image, the foreground is characterized by very high values. An example radiography image containing foreground and background is presented in Fig. 3 .

The aforementioned regions are not of interest in the diagnostic process, which leads to the conclusion that their enhancement would not bring any benefit. Thus, they should be detected and disregarded by the processing algorithm. Background detection and subsequent elimination can be performed by utilizing algorithms for collimation field detection [20]-[25].

Absolute values of coefficients of $L P_{1}$ for image shown in Fig. 3 are presented in Fig. 4. It is visible that coefficients corresponding to foreground have lower values than the ones corresponding to the anatomy or to the image background.

Coefficients of the first three layers of the LP for the central horizontal profile of the example image shown in Fig. 3 are presented in Fig. 5. The topmost graphic represents pixel values, while the rest represent LP coefficients for respective layers. Note that LP graphics do not show absolute values of the coefficients, as the central value of the ordinate corresponds to zero, with ordinate values omitted since they are dependent on pixel values which vary image-wise.

LP coefficients which correspond to foreground (highest values in the topmost graphic of Fig. 5) have values near zero for all of the three layers, that is, they are similar for different scales. It should be noted that LP coefficients represent image details of different scales and that coefficients of lower LP layers correspond to finer details, while coefficients of higher LP layers correspond to coarser details. From this we conclude that detection of low-valued details (LVD) can be achieved by applying a threshold on corresponding higher layer LP coefficients. The first step is the detection of LVDs on a higher layer: 


$$
M_{n}=\left|L P_{n}\right|<\mathrm{T}
$$

where $\mathrm{T}$ denotes the threshold, $|\cdot|$ is absolute value operator, while $M_{n}$ stands for the binary mask of LVDs on LP layer marked with $n$. Higher layer LVDs can be detected through nearest neighbor interpolation of $M_{n}$. Similar LP coefficients correlation was used for noise reduction in [10]. Similar analysis paired with wavelet transformation was used for image denoising in [11] and [12].
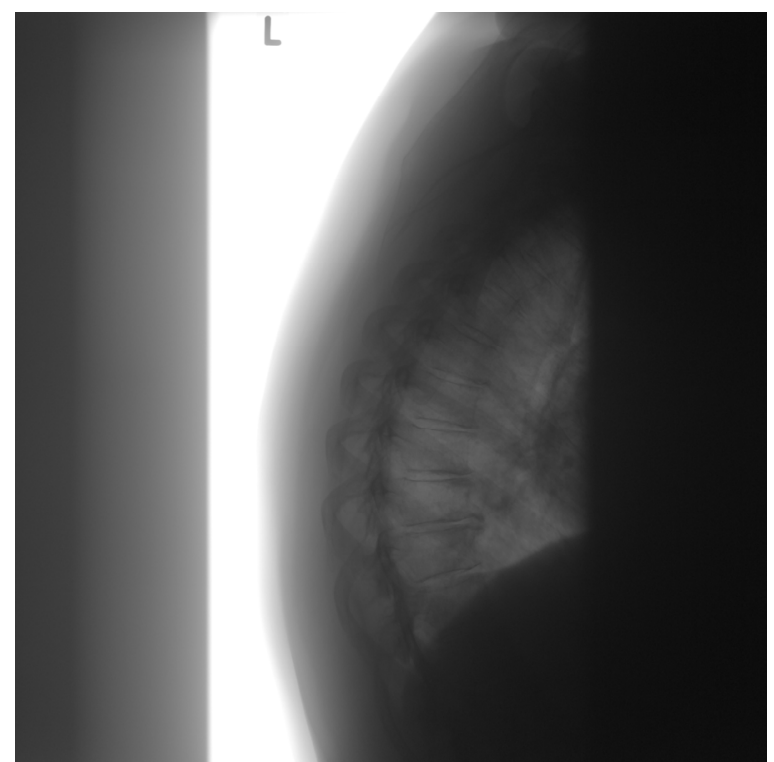

Fig. 3. Example thoracic spine image with linear to logarithmic domain conversion applied for better visibility. Background is visible on the left side of the image (low-value region). Foreground is visible in the center of the image (high-value region).

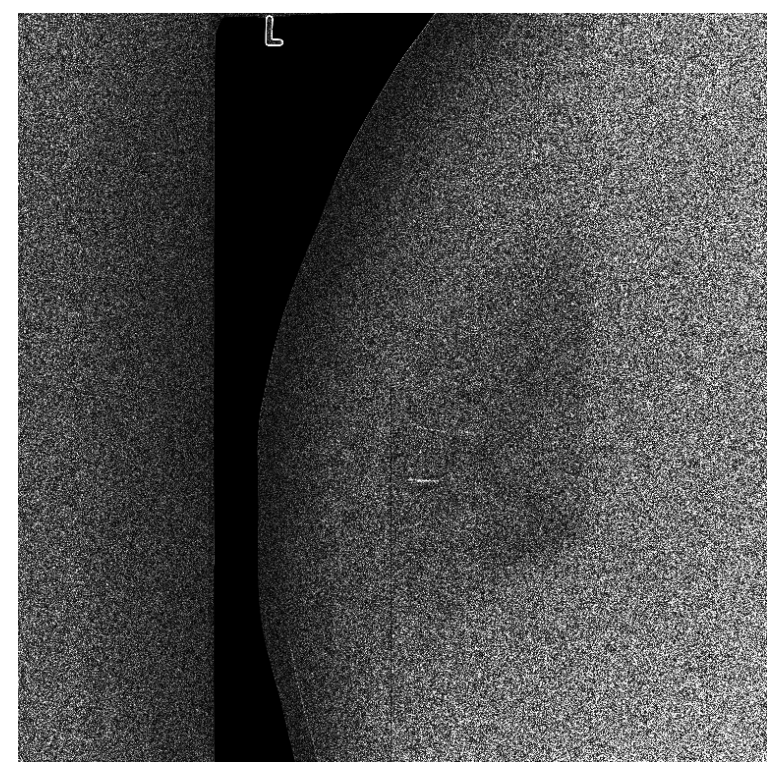

Fig. 4. Absolute values of coefficients of $L P_{1}$ obtained by decomposing image shown in Fig. 3.

LVDs in $L P_{1}$, detected based on the binary mask $M_{3}$, are shown as binary mask in Fig. 6. In this example threshold $\mathrm{T}$ was set to 0.00001 of the pixel value range. Analysis of the threshold selection is presented in Section VII. It is determined that, with respect to the used threshold, $11.13 \%$ of the example image are LVDs, corresponding to the amount of pixels which can be disregarded by the processing algorithm and should even be omitted in the LP calculation process.

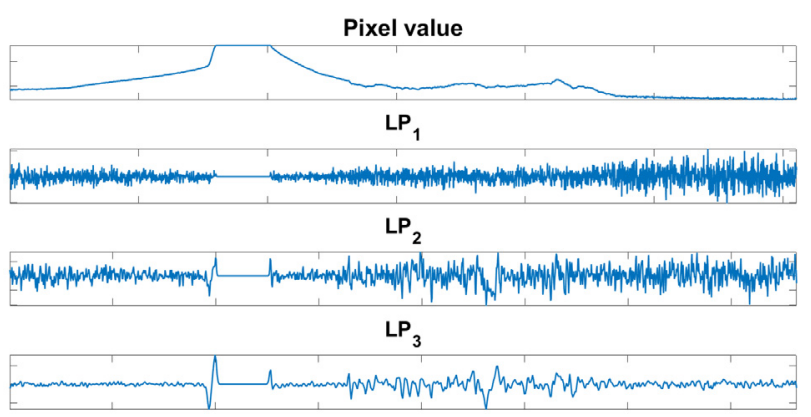

Fig. 5 Pixel values and coefficients on corresponding LP layers for the central horizontal profile of the example image shown in Fig. 3.

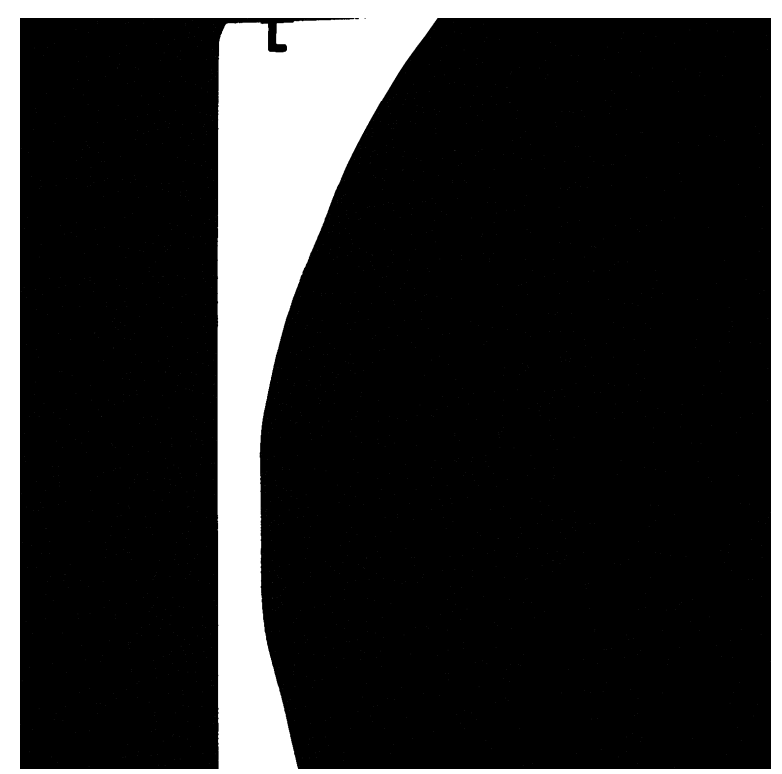

Fig. 6. Binary mask of LVDs in $L P_{1}$ detected based on the binary mask $M_{3}$.

\section{LAPLACIAN PYRAMID MODIFICATION}

Method presented in Section IV uses higher LP layers for detection of LVDs on lower ones. In order to modify the LP calculation using the LVD detection with the aim of processing time reduction, LP should be calculated in an inverse manner, i.e. higher layers prior to lower ones. Based on the observation of algorithm presented in Fig. 1, we propose Gaussian pyramid calculation prior to LP calculation according to the algorithm presented in Fig. 7.

$$
\begin{gathered}
\text { Input } \\
G P_{0} \leftarrow \text { input image } I \\
\text { For } i=0 \ldots N-1 \\
G P_{i+1}=\operatorname{convolution}\left(G P_{i}, \operatorname{lp} f\right) \\
G P_{i+1}=\operatorname{subsample}\left(G P_{i+1}\right) \\
\text { end } \\
\text { Res }=G P_{N}
\end{gathered}
$$

Fig. 7. Gaussian pyramid calculation algorithm. 
Algorithm shown in Fig. 7 is created by omitting the steps needed for LP calculation as the Gaussian pyramid is independent of it. One LP layer calculation needs two Gaussian pyramid layers, the current and the next one. Layer $L P_{i}$ is determined through subtraction of $\widehat{G P}_{i}$, which is obtained by upsampling and low-pass filtering of $G P_{i+1}$, from $G P_{i}$. This can be done in an inverse manner when the Gaussian pyramid layers are previously determined, as shown in Fig. 8:

$$
\begin{gathered}
\text { For } i=N-1 \ldots 0 \\
\widehat{G P}_{i}=\text { upsample }\left(G P_{i+1}\right) \\
\widehat{G P}_{i}=\operatorname{convolution}\left(\widehat{G P}_{i}, l p f\right) \\
L P_{i}=G P_{i}-\widehat{G P}_{i} \\
\text { end }
\end{gathered}
$$

Fig. 8. LP layer calculation in the inverse manner.

Subtraction in the algorithm shown in Fig. 8 is performed element-wise between $G P_{i}$ and $\widehat{G P}_{i}$, thus for calculating a single coefficient $L P_{i}(x, y)$ one needs to know only the values of $G P_{i}(x, y)$ and $\widehat{G P}_{i}(x, y)$ ( $x$ and $y$ are spatial coordinates). $G P_{i}(x, y)$ values are already known from the Gaussian pyramid, thus only the $\widehat{G P}_{i}(x, y)$ should be determined. This value can be obtained by upsampling and filtering only the corresponding part of the $G P_{i+1}$. This leads to the conclusion that upsampling the whole layer $G P_{i+1}$ is not required if not all of the $\widehat{G P}_{i}$ values are going to be used. As lower layer LVDs can be detected based on higher layer LVDs, calculation of $\operatorname{LVD} L P_{i}(x, y)$ can be omitted by setting its value to zero, hence corresponding $\widehat{G P}_{i}(x, y)$ should not be calculated. Algorithm which incorporates previous observations in the LP calculation is presented in Fig. 9:

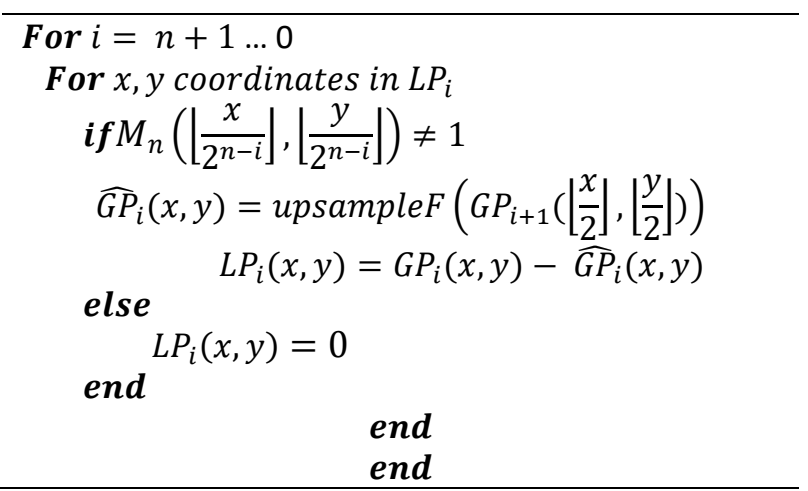

Fig. 9. LP calculation based on LVD detection.

where $M_{n}$ stands for the binary mask of LVDs on LP layer marked with $n,\lfloor\cdot]$ represents rounding to the nearest integer towards minus infinity whileupsampleFdenotes upsampling and filtering. Previously explained procedure contributes to processing time reduction in two ways. Firstly, for layer $L P_{i}, i>n$, calculation is reduced by the percentage of the LVDs in that layer. Secondly, radiography image enhancement algorithms can omit the LVDs as they do not contribute to the image diagnostic value. What is more, it will reduce noise as coefficients with a low value are estimated as noise and omitted in the enhancement (see Section II).

\section{PROCESSING TIME REDUCTION ANALYSIS}

Complexity of the radiography image processing algorithm used in this paper is linearly proportional to the number of coefficients in the LP, $P$, which can be calculated as follows:

$$
\begin{aligned}
P=\sum_{i=0}^{N} \operatorname{size}\left(L P_{i}\right) & =\sum_{i=0}^{N} \frac{1}{4^{i}} \operatorname{size}(I)= \\
& =\operatorname{size}(I) \sum_{i=0}^{N} \frac{1}{4^{i}}
\end{aligned}
$$

where operator size $(\cdot)$ determines the number of pixels in an image, the input image is denoted as $I$ and $N$ is the number of the pyramid layers. It should be noted that the supremum of $P$, calculated when $N \rightarrow \infty$ is $\frac{4}{3} \operatorname{size}(I)$.

$L P_{1}$ has the same number of pixels (coefficients) as $I$, thus contributes with $75 \%$ to the overall number of coefficients $P$. As the number of coefficients in $L P_{2}$ is $1 / 4$ of size $(I)$, it contributes to $P$ with $18.75 \%$. Therefore, $93.75 \%$ of the processing time is used for the first two LP layers as the complexity of the processing algorithm is linearly proportional to $P$. We conclude that most of the processing time reduction gained by using LVD detection can be achieved on the first two LP layers. In our experiments, $L V D$ detections for $L P_{1}$ and $L P_{2}$ were based on coefficient values in $\mathrm{LP}_{3}$.

\section{EVALUATION AND RESULTS}

For evaluation of the proposed approach a database consisting of 47 clinical digital radiography images was employed. The database consists of images of a wide range of anatomies (spine, limbs, head, chest, etc.) of different patients, acquired during standard daily hospital routine. Varian PaxScan 4343 flat panel detector was used in the image acquisition process. Detector pixel is squared and $139 \times 139 \mu \mathrm{m}$ in size. Obtained images are of size $3072 \times$ 3072 pixels.

For the purpose of determining the amount of coefficients in $L P_{1}$ which are estimated as LVDs we varied the value of $\mathrm{T}$, defined in (1), in the range from 0 to 0.02 of the input image value range. Note that the portion of $L P_{2}$ detected as LVDs is the same as that of $L P_{1}$, as both detections were based on $M_{3}$.

The mean value of the portion of $L P_{1}$ detected as LVDs depending on the value of $\mathrm{T}$, measured on the entire evaluation database, is presented in Fig. 10.

From observing the presented figure, several conclusions can be deduced. Firstly, the portion of coefficients estimated as LVDs increases with the value of $\mathrm{T}$, which could be expected considering that LP layers have a zero mean value. Even for very low values of $\mathrm{T}$ a high portion of coefficients will be detected as LVDs, suggesting that using high values for $\mathrm{T}$ might lead to fine detail loss as they are represented through lower LP layers (see Section III). Finally, the estimate does not vary significantly through the database as can be seen from the error bars. 


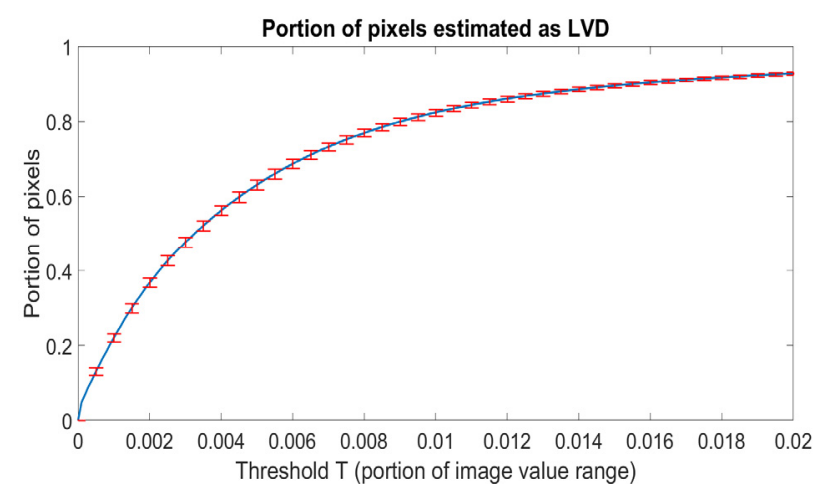

Fig. 10. Portion of $L P_{1}$ detected as LVD.

In order to evaluate the effect of value of $\mathrm{T}$ on image quality, we compared images processed by the enhancement algorithm with and without embedded LVD detection. It should be noted that the images were processed after the removal of the background. To measure the similarity between the obtained images we employed the structural similarity index (SSIM). For two images $A$ and $B$, with the same dimensions and pixel number $M$, SSIM is defined as:

$$
\begin{aligned}
& \operatorname{SSIM}(A, B) \\
& =\frac{1}{M} \sum_{x, y} \frac{\left(2 \mu_{A} \mu_{B}+c_{1}\right)\left(2 \sigma_{A B}+c_{2}\right)}{\left(\mu_{A}^{2}+\mu_{B}^{2}+c_{1}\right)\left(\sigma_{A}^{2}+\sigma_{B}^{2}+c_{2}\right)}
\end{aligned}
$$

where $\mu_{A}, \mu_{B}, \sigma_{A}, \sigma_{B}$ and $\sigma_{A B}$ are the local means, standard deviations and cross-covariance calculated in the neighborhoods of $A(x, y) \operatorname{and} B(x, y)$, while $c_{1}$ and $c_{2}$ represent calculation constants [26].

Dependence of mean SSIM on the value of T, obtained on the entire database, is presented in Fig. 11. The graphic shows the decrease of mean SSIM with the increase of the value of $\mathrm{T}$, which is contrary to the portion of coefficients estimated as LVDs. This is in accordance with the increase of estimated LVDs as more fine details will be set to a zero value.

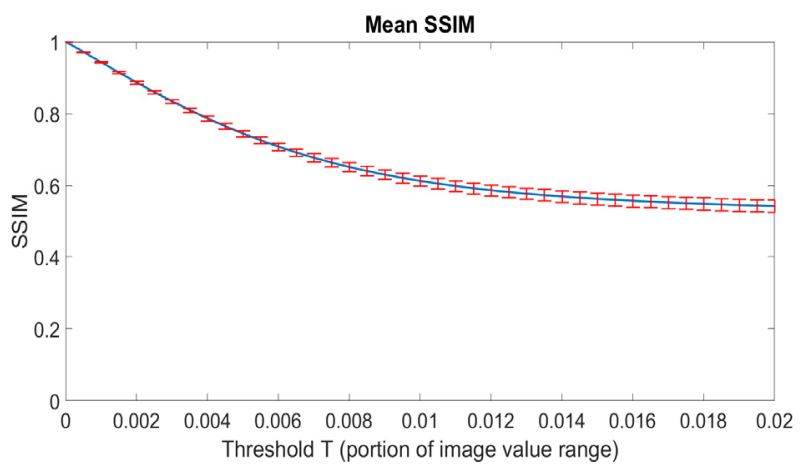

Fig. 11. Mean SSIM value dependence on the value of threshold T.

To achieve a compromise between decreasing processing time and maintaining image quality, a threshold should be determined so that it simultaneously corresponds to both a high portion of coefficients estimated as LVDs and a high
SSIM value. Relation of the mean SSIM value and portion estimated as LVDs for the same values of threshold $\mathrm{T}$ is shown in Fig. 12. It can be seen that high SSIM values are obtained only when a small portion of the fine details is estimated as LVDs. Nevertheless, even with the threshold $\mathrm{T}$ set to the 0.0001 of the image value range mean processing time will be reduced by $4.62 \%$, while achieving a mean SSIM value of 0.995 .

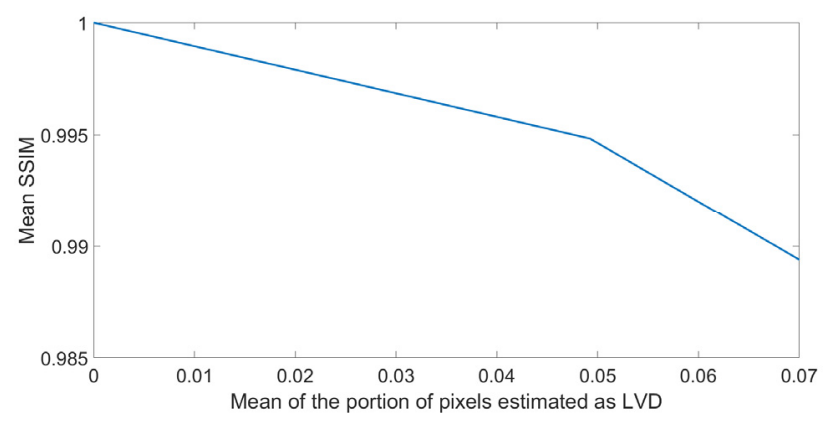

Fig. 12. Relation between SSIM and portion of fine details detected as LVDs.

\section{CONCLUSION}

In this paper we proposed a thresholding approach to radiography image processing acceleration. The approach is based on detecting low-value Laplacian pyramid coefficients which can be omitted by the radiography image enhancement algorithm. The detection is based on correlation between coefficients on different layers of the Laplacian pyramid. Low-value coefficients which correspond to finer details (lower pyramid layers) were detected based on low-value coefficients which correspond to coarser details (higher pyramid layers) and are lower than the threshold.

The detection of low-value details was embedded in the multi-scale radiography image enhancement algorithm. Relation between obtained image quality and the portion of fine details which were estimated as low-valued was analysed. The quality of images obtained with the proposed approach is shown to depend on the threshold used for the detection.

Setting the threshold value to 0.0001 of the image value range results in processing time reduction of $4.62 \%$. This threshold value was chosen as it presents a compromise between processed image quality and processing time reduction, since it results in mean SSIM value of 0.995 with respect to images processed without the detection.

\section{REFERENCES}

[1] P. Vuylsteke, and E. Schoeters, "Multiscale Image Contrast Amplification", in SPIE Image Processing, vol. 2167, pp. 551-560, 1994.

[2] M. Stahl, T. Aach, T. M. Buzug, S. Dippel, U. Neitzel, "Noiseresistant weak-structure enhancement for digital radiography," in Proc. SPIE Medical Imaging 1999: Image Processing, San Diego, USA, vol. 3661, pp. 1406-1417, February 20-26, 1999.

[3] XuanqinMou and Min Zhang, "Nonlinear multi-scale contrast enhancement for chest radiograph," 2008 15th IEEE International Conference on Image Processing, San Diego, CA, 2008, pp. 3184-3187. doi: 10.1109/ICIP.2008.4712472.

[4] A. Laine, J. Fan, and W. Yang, "Wavelets for Contrast Enhancement of Digital Mammography," IEEE Eng. Med. Biol. Mag., vol. 14 pp.536-550, 1995. 
[5] J. P. Bolet, A. R. Cowen, J. Launders, J. Davies, G. J. S. Parkin, and R. F. Bury, "Progress with an "all-wavelet" approach to image enhancement and de-noising of direct digital thorax radiographic images," in 1997 Sixth International Conference on Image Processing and Its Applications, vol. 1, pp. 244-248, Jul 1997.

[6] S. Dippel, M. Stahl, R. Wiemker, and T. Blaffert, "Multiscale Contrast Enhancement for Radiographies: Laplacian Pyramid Versus Fast Wavelet Transform", IEEE Trans. Med. Imag., vol. 21, no. 4, pp. 343-353, April 2002.

[7] Y. Derong, Z. Yuanyuan and L. Dongguo, "Fast Computation of Multiscale Morphological Operations for Local Contrast Enhancement," 2005 IEEE Engineering in Medicine and Biology 27th Annual Conference, Shanghai, 2005, pp. 3090-3092. doi: 10.1109/IEMBS.2005.1617128.

[8] Y. Matviychuk et al., "Learning a multiscale patch-based representation for image denoising in X-RAY fluoroscopy," 2016 IEEE International Conference on Image Processing (ICIP), Phoenix, AZ, USA, 2016, pp. 2330-2334. doi: 10.1109/ICIP.2016.7532775

[9] P. J. Burt, and E. H. Adelson, "The Laplacian Pyramid as a Compact Image Code", IEEE Trans. on communications, Vol. Com-31, no. 4, April 1983.

[10] M. Hensel, U. Brummund, T. Pralow, R. R. Grigat, "Noise Reduction with Edge Preservation by Multiscale Analysis of Medical X-Ray Image Sequences," in Bildverarbeitungfür die Medizin 2005: Algorithmen - Systeme - Anwendungen Proceedings des Workshops vom 13. - 15. März 2005 in Heidelberg, H. P. Meinzer, H. Handels, A. Horsch, T. Tolxdorff, Ed. Berlin: Springer Berlin Heidelberg, 2005, pp. 55-59. doi: 10.1007/3-540-26431-0 12

[11] A. Pizurica, W. Philips, I. Lemahieu and M. Acheroy, "Ā versatile wavelet domain noise filtration technique for medical imaging," in IEEE Transactions on Medical Imaging, vol. 22, no. 3, pp. 323-331, March 2003. doi: 10.1109/TMI.2003.809588

[12] Paul Bao and Lei Zhang, "Noise reduction for magnetic resonance images via adaptive multiscale products thresholding," in IEEE Transactions on Medical Imaging, vol. 22, no. 9, pp. 1089-1099, Sept. 2003. doi: 10.1109/TMI.2003.816958

[13] E. L. Nickoloff, "Aapm/rsna physics tutorial for residents: Physics of flat-panel fluoroscopy systems," RadioGraphics, vol. 31, no. 2, pp. 591-602, 2011, pMID: 21415199. [Online]. Available: http://dx.doi.org/10.1148/rg.312105185

[14] M. J. Yaffe, "Detectors for digital mammography," in Digital Mammography, U. Bick and F. Diekmann, Eds. Berlin, Heidelberg: Springer Berlin Heidelberg, 2010, pp. 13-31. [Online]. Available: http://dx.doi.org/10.1007/978-3-540-78450-0 2

[15] V. Ostojić, Đ. Starčević and V. Petrović, "Low-value Laplacian pyramid coefficient prediction for faster radiography image processing," 2016 24th Telecommunications Forum (TELFOR), pp. 1-4, 2016. doi: 10.1109/TELFOR.2016.7818803

[16] V. Ostojić, Đ Starčević and V. Petrović, "Artifact reduction in multiscale contrast enhancement for digital radiography," Telecommunications Forum Telfor (TELFOR), 2014 22nd, Belgrade, 2014, pp. 513-516. doi: 10.1109/TELFOR.2014.7034459

[17] P. J. Burt, "Fast filter transform for image processing," Computer Graphics and Image Processing, vol. 16, no. 1, pp. 20 - 51, 1981

[18] P. J. Burt, "Fast algorithms for estimating local image properties," Computer Vision, Graphics, and Image Processing, vol. 21, no. 3, pp. 368-382, 1983.

[19] R. A. Haddad and A. N. Akansu, "A class of fast gaussian binomial filters for speech and image processing," IEEE Transactions on Signal Processing, vol. 39, no. 3, pp. 723-727, Mar 1991.

[20] J. Zhang and H. K. Huang, "Automatic Background Recognition and Removal (ABRR) in Computed Radiography Images" IEEE Trans. Medical Imaging, vol. 16, no. 6, pp. 762-771, Dec. 1997

[21] R. Wiemker, S. Dippel, M. Stahl, T. Blaffert and U. Mahlmeister, "Automated recognition of the collimation field in digital radiography images by maximization of the Laplace area integral" Proc. SPIE, Medical Imaging 2000: Image Processing, vol. 3979, pp. $1555-1565,2000$

[22] T. M. Lehmann, S. Goudarzi, N. Linnenbrugger, D. Keysers and B. B. Wein, "Automated localization and delineation of collimation fields in digital and film-based radiographs" Proc. SPIE, Medical Imaging 2002: Image Processing, vol. 4684, pp. 1215-1223, 2002

[23] I. Kawashita, M. Aoyama, T. Kajiyama and N. Asada, "Collimation Detection in Digital Radiographs Using Plane Detection Hough Transform" in Knowledge-Based Intelligent Information and Engineering Systems, 1 st ed. vol. 2774, V. Palade, Ed., Springer-Verlag Berlin Heidelberg, 2003, pp. 394-401 [7th International Conference, KES 2003 Oxford, UK, September 3-5, 2003 Proceedings, Part II]

[24] H. Mao, Z. Peng, F. Dennerlein, Y. Shinagawa, Y. Zhan and X. S. Zhou, "Multi-view learning based robust collimation detection in digital radiographs" Proc. SPIE, Medical Imaging 2014: Image Processing, vol. 9034, pp. 903421-1 - 903421-6, 2014

[25] V. Ostojić, Đ Starčević and V. Petrović, "Detection of collimation field in digital radiography using Frobenius norm of Hessian," Telecommunications Forum Telfor (TELFOR), 2015 23rd, Belgrade, 2015, pp. 476-479. doi: 10.1109/TELFOR.2015.7377510

[26] Z. Wang, A. C. Bovik, H. R. Sheikh and E. P. Simoncelli, "Image quality assessment: from error visibility to structural similarity," in IEEE Transactions on Image Processing, vol. 13, no. 4, pp. 600-612, April 2004. doi: 10.1109/TIP.2003.819861 